\title{
Prenatal Population Screening for Fragile X Carrier and the Prevalence of Premutation Carriers in Korea
}

\author{
Sung-Hee Han, Yun-Ah Heo, Young-Ho Yang, Young-Jin Kim, Han-lk Cho and Kyoung-Ryul Lee* \\ Division of Molecular Genetics, Department of Laboratory Medicine, Seoul Medical Science Institute, Seoul Clinical Laboratories, Seoul, Korea
}

\begin{abstract}
Purpose: Fragile X carrier detection before or at early pregnancy through a wide screening program may not only confer a risk of having offspring with Fragile X syndrome (FXS), but may also confer a risk for Fragile X-associated primary ovarian insufficiency and Fragile $X$-associated tremor/ataxia syndrome. However, prior to the implementation of such a program, the carrier prevalence in a population and the availability of effective screening test should be evaluated. The aim of our study was to determine the prevalence of premutation carriers and to evaluate the feasibility of screening test.

Materials and Methods: The blood samples were obtained from 8,641 pregnant women with no family history of mental retardation. We performed a three-primer CGG repeat primed (RP) PCR using the AmplideX ${ }^{\text {TM }}$ FMR1 PCR kit (Asuragen, Inc. Austin, TX, USA). Samples showing full mutation alleles were reflexed to Southern blot analysis for methylation status and sizing.

Results: Among the 8,641 women, we found 8 premutation carriers (1:1,090, 0.09\%) and 46 women with an intermediate allele $(1: 190,0.53 \%)$. No woman was found to carry the fully mutated allele. All the detected alleles were within the CGG repeat range of 8-117. Among the 8,641 samples, 29 and 30 CGG repeats represent $66.6 \%$ of all cases. The CGG RP PCR method provides robust detection of expanded alleles and resolves allele zygosity, thus minimizing the number of samples that require Southern blot analysis.

Conclusion: This is the first study that has focused on the prevalence of FXS premutation carriers and FMR1 allele distribution in normal pregnant women. These data have important implications for population-based fragile X carrier screening in Korea.
\end{abstract}

Key words: Fragile X syndrome, FMR1 premutation carrier, FMR1 allele distribution, Population screening

\section{Introduction}

FragileXsyndrome(FXS,0MIM300624) iscaused by theexpansion of the CGG repeat in the $5^{\prime}$ untranslated region (UTR) of the FMR1 (FragileX mental retardation 1) gene located on the X chromosome." Smaller expansions (55-200 CGG repeats; premutation) of the FMR1 gene are not associated with intellectual disability, but do convey increased risk for Fragile X-associated primary ovarian insufficiency (FXPOI) and Fragile X-associated tremor/ataxia syndrome (FXTAS). ${ }^{2)}$
Because of potential repeat instability upon the transmission of premutation alleles, healthy women with alleles in this range are considered to be at risk of having children affected with FXS. ${ }^{3)}$ Also, women identified as carriers of premutation alleles should be counseled as to the risk of ovarian dysfunction, the reproductive implications, and the reproductive options available to them. Thus, screening women in their early reproductive years for a possible genetic predisposition to a higher rate of reproductive failure might allow them to monitor their ovarian function early enough to better

Received: 08 Nov 2012, Revised: 20 Dec 2012, Accepted: 22 Dec 2012, Published: 31 Dec 2012

*Corresponding author: Kyoung-Ryul Lee, M.D., Ph.D.

Department of Laboratory Medicine Seoul Medical Science Institute, Seoul Clinical Laboratories 7-14 Dongbinggo-dong, Yongsan-gu, Seoul, Korea Tel: +82-2-330-0510, Fax: +82-2-790-6509, E-mail: dkrlee@scllab.co.kr

(c) This is an open-access article distributed under the terms of the Creative Commons Attribution Non-Commercial License (http://creativecommons.org/licenses/by-nc/3.0/) which permits unrestricted non-commercial use, distribution, and reproduction in any medium, provided the original work is properly cited.

(c) Copyright 2012 by the Korean Society of Medical Genetics

www.e-kjgm.org 
achieve their reproductive goals. ${ }^{3)}$ Before the implementation of a population-based screening program is considered, the prevalence of carrier women in a population should first be determined. For premutation alleles, allele frequencies are more often estimated through general population screening. In order to implement routine population-based FXS carrier screening in Korea, a pilot screening process must be initiated to estimate the female carrier rate in general Korean women population.

Until recently, however, the technical difficulties of performing population-based carrier detection precluded offering screening to the general population. As large expansions are refractory to PCR amplification, strategies for prenatal population-based carrier screening for pregnant women required both a PCR and Southern blot analysis. Combined, these are costly and laborintensive, have prolonged turnaround times, and ultimately have limited utility for population-based screening. Recent studies have described a method, CGG repeat primed PCR (CGG RP PCR) coupled with capillary electrophoresis, that overcomes the technical hurdles to implementing carrier screening and that has the potential to be used for population-based screening. In this study, using CGG RP PCR by reducing the number of Southern blot analyses, we determined the FMR1 premutation carrier frequencyin prenatal population-based FragileXcarrierscreening for pregnant women in Korea.

\section{Material and Methods}

A total of 8,641 pregnant women applied for testing on their own initiative or on the advice of their physicians, on a self-pay basis, from various medical sites in Korea. Institutional review board (IRB) approval was obtained for the study from our IRB committee. They were given printed information on fragile $X$ and each completed a questionnaire to ascertain any family history of mental retardation, and all women with such a family history were excluded from the study. All the women who were found to be carriers of premutation alleles were offered genetic counseling. They were provided with information about prenatal diagnosis based on amniotic fluid analysis or chorionic villus sampling (CVS). Genomic DNA was isolated from peripheral blood leukocytes (5 $\mathrm{mL}$ of whole blood) using a standard method (QIAamp DNA Blood Mini kit; Qiagen, Hilden, Germany).

We performed the CGG RP PCR using an AmplideX ${ }^{\mathrm{TM}}$ FMR1 PCR kit (Asuragen, Inc. Austin, TX, USA) to determine the number of $C G G$ repeats in the FMR1 gene using PCR and fragment sizing by capillary electrophoresis (CE). Samples showing full mutation alleles were reflexed to Southern blot analysis for methylation status and sizing. Southern blot analysis was performed as described. ${ }^{5)}$ The preparation and analysis of CGG RP PCR was done according to the kit instructions. PCR was performed on an ABI GeneAmp PCR system 9700 thermal cycler (Applied Biosystems, Foster City, CA, USA). All amplicons were sized on an ABI 3130xI Genetic Analyzer (Applied Biosystems) and PCR products detected by CE were analyzed using GeneMapper 4.0 software (Applied Biosystems). The FMR1 alleles were categorized according to the American College of Medical Genetics guidelines for normal ( $<45$ CGG repeats), intermediate (45-54 CGG repeats), premutation (55-200 CGG repeats), and full mutation (>200 CGG repeats) alleles.

\section{Results}

Among 8,641 pregnant women screened for FMR1 allelic expansion, we found 8 carriers of an allele with $>55$ repeats, representing a premutation carrier frequency of 1:1,090 (0.09\%). No woman was found to carry the fully mutated allele. The mosaic alleles were not detected in any cases, including premutation carriers. Table 1 details the number of premutation carriers for each range of the number of CGG repeats. Compared with the premutation frequency of normal women in Caucasian populations (Table 2), this proved to be much lower than that of Israel, USA, and Finland. ${ }^{7-13)}$

A total of 44 different $C G G$ repeat alleles ranging in number from 8-117 were present. Asseen in Fig. 1, the most common allele was 29 repeats (37.1\%), followed by 30 repeats (29.5\%). Among the 8,641 samples, 29 and 30 CGG repeats represent $66.6 \%$ of all cases. Besides this major peak, there was a second peak with 35 and 36 repeats (Fig. 1). The overall normal allele prevalence was $99.5 \%$ $(8,598 / 8,641)$. Intermediate size was defined as $45-54$ repeats; a total of 46 women were found to be within this range, with an intermediate allele prevalence of 1:190 (0.53\%).

Table 1. Number of Premutation Carriers for Each Range of the Number of CGG Repeats

\begin{tabular}{lc}
\hline CGG repeat range & No. of women $(n=8)$ \\
\hline $55-60$ & 2 \\
$61-65$ & 1 \\
$66-70$ & 2 \\
$71-75$ & 1 \\
$76-80$ & 0 \\
$81-200$ & 2 \\
$>200$ & 0 \\
\hline
\end{tabular}


Table 2. Carrier Frequency of FMR1 Premutation in Prenatal Population-based Fragile X Screening among Different Ethnic Populations

\begin{tabular}{|c|c|c|c|c|}
\hline Populations & Samples screened & Premutation identified & Carrier Frequency (\%) & References \\
\hline Israel-1 & $8,426^{a}$ & 58 & $1: 143(0.70)$ & Pesso et al. ${ }^{7)}$ \\
\hline Israel-2 & $14,334^{\mathrm{a}}$ & 127 & $1: 113(0.88)$ & Toledano-Alhadef et al. \\
\hline Israel-3 & $36,483^{\mathrm{a}}$ & 231 & 1: $158(0.63)$ & Berkenstadt et al.9) \\
\hline USA-1 & $474^{\mathrm{a}}$ & 3 & $1: 158(0.63)$ & Spence et al. ${ }^{10)}$ \\
\hline USA-2 & $2,299^{a}$ & 6 & $1: 382(0.26)$ & Cronister et al. ${ }^{11)}$ \\
\hline Finland-1 & $1,477^{b}$ & 6 & $1: 246(0.41)$ & Ryynanen et al. ${ }^{12)}$ \\
\hline Finland-2 & $220^{b}$ & 1 & $1: 220(0.45)$ & Kallinen et al. ${ }^{13)}$ \\
\hline Korea & $8,641^{a}$ & 8 & $1: 1,090(0.09)$ & Present study \\
\hline
\end{tabular}

${ }^{a}$ Pregnant or non-pregnant women without a family history of mental retardation

bregnant or non-pregnant women with no information about a family history of mental retardation

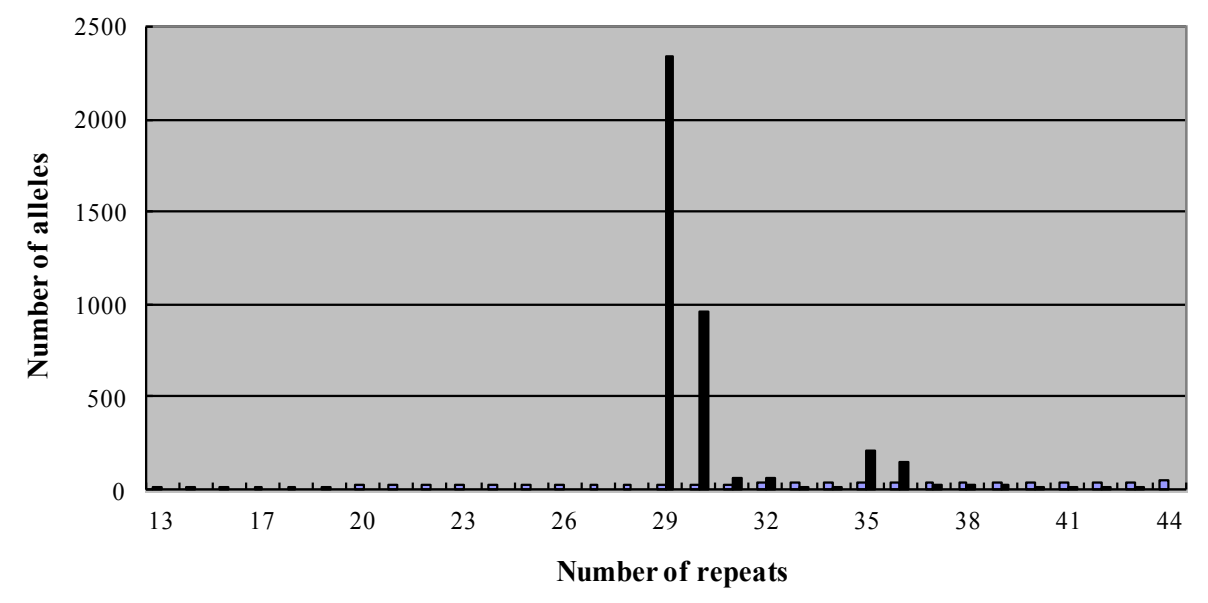

Fig. 1. Allele frequencies for CGG repeats in FMR1 in 8,641normal pregnant Korean women.

\section{Discussion}

Despite recent interest in widespread carrier screening for FMR1 mutations in prenatal settings, current consensus guidelines from multiple professional organizations (American Congress of Obstetricians and Gynecologists, American College of Medical Genetics, National Society of Genetic Counselors) have not recommended universal carrier testing. ${ }^{3)}$ However, there appears to growing interest in more liberal screening for Fragile Xamong certain educated patient groups and medical professionals. Indeed, commercial laboratories have begun marketing Fragile $X$ carrier testing to prenatal pregnant women through educational pamphlets supplied to obstetric and prenatal diagnosis clinics and offices, especially in Korea. Our data demonstrated that if population-based carrier detection for FXS were introduced in Korea, 1:1,090 (0.09\%) women would be determined to be at risk for conceiving children with FXS or related syndromes. This number is much lower than in Caucasian populations for prenatal FXS carrier screening tests, as shown in Table 2. The prevalence of having an FXS fetus and FXPOI in Korean women was assumed to be not reasonably high.

Although mutation frequencies were reported in all studies, it is difficult to make comparisons between studies as different repeat length cutoffs, sample sizes, and sampling methods have been used. Consistency in repeat length cutoffs and large studies with a wide range of population groups are needed. ${ }^{14)}$ While the lack of any premutation alleles has been reported in other Asian studies in Japan and Taiwan, as well as in a previous Korean study, ${ }^{15-17)}$ the number of collected samples in those studies was much fewer than the number of samples collected in this study. This is the first report to conclusively demonstrate the premutation carrier frequency in a general population in Korea. According to a report by Musci et al., a prenatal Fragile X carrier screening program that aims to screen all pregnant women regardless of family history or specific risk factors, would be cost effective based on a reasonably wide range of assumptions. ${ }^{3)}$ Considering the very low premutation frequency in our data, we doubt such a wide screening program in Korea would be cost-effective or appropriate. However, arguments in favor of introducing population-based screening for FXS center on the severity of the 
condition and its impact on individuals, families, and society., ${ }^{3,14)}$

The intermediate alleles have been shown to be slightly unstable upon transmission. The American College of Medical Genetics has recommended that intermediate alleles be considered a possible risk factor for repeat expansion. ${ }^{6}$ Here, we estimated the intermediate allele prevalence was 1:190 females. This frequency was lower than the intermediate allele frequencies reported in previous reports for Caucasian populations. ${ }^{14)}$

In previous studies on the variation of the CGG repeat in the FMR1 gene, the CGG repeat allele frequencies differed in terms of ethnicity. In FMR1, the 30 CGG repeat allele was the most common, followed by 29 in Western European ancestry populations. ${ }^{18)}$ The present study of FMR1 CGG repeats in a normal Korean population indicates the 29 and 30 CGG repeats are most commonly observed, representing $66.6 \%$ of all cases. However, the 29 alleles (37.1\%) present at a higher frequency than the 30 alleles (29.5\%), which is similar to other Asian populations. ${ }^{18)}$ In the present study, the distribution of repeat size in our Korean population is skewed to the smaller size, which may indicate that the chance of premutation occurring in our population is lower than in Western European ancestry populations.

PCR amplification of the $C G G$ repeats region in FMR1 in combination with Southern blotting are the most commonly used techniques for CGG repeat detection and sizing. ${ }^{19,20)}$ PCR can easily detect normal alleles and most premutations; however, amplification of large premutations and full mutations is technically challenging. This is particularly difficult when working with specimens from females. FMR1 Southern blot analysis is used both to characterize samples with numbers of CGG repeats too large to amplify by PCR and to determine the methylation status of the gene. ${ }^{19,20)}$ Unfortunately, this workflow is costly, time- and laborintensive, and requires large amounts of genomic DNA, making it unsuitable for higher testing volumes or population screening. We incorporated the CGG RP PCR method and significantly reduced the number of Southern blot analyses required as the method can be used to distinguish normal homozygote females from full mutation carrier females, which in our experience represent $>99$ $\%$ of all samples from full mutation carriers. This method detects all carriers of premutations and full mutations, both male and female, including mosaic carriers. Previous studies show that the CGG RP PCR method provides robust detection of expanded alleles and resolves allele zygosity, thus minimizing the number of samples that require Southern blot analysis and producing more comprehensive FMR1 genotyping data than other methods. ${ }^{4,211}$

In conclusion, we demonstrated that the premutation carrier frequency of FXS in the normal pregnant Korean women is
1:1,090, which is much lower than in Western countries. It is now assumed that the prevalence of FXPOI is low in Korea. Several limitations are noted in our present study, mainly due to its subject enrollment. Regardless of these limitations, however, our data could offer groundwork for a proper prenatal population-based Fragile $X$ carrier screening program for reproductive women in Korea.

\section{References}

1. Verkerk AJ, Pieretti $M$, Sutcliffe JS, Fu YH, Kuhl DP, Pizzuti $A$, et al Warren Identification of a gene (FMR-1) containing a CGG repeat coincident with a breakpoint cluster region exhibiting length variation in fragile X syndrome. Cell 1991;65:905-14.

2. Jacquemont $S$, Hagerman RJ, Hagerman PJ, Leehey MA. Fragile-X syndrome and fragile $X$-associated tremor/ataxia syndrome: two faces of FMR1. Lancet Neurol 2007;6:45-55.

3. Musci TJ, Moyer K. Prenatal carrier testing for fragile $X$ : counseling issues and challenges. Obstet Gynecol Clin North Am 2010;37:61-70.

4. Filipovic-Sadic S, Sah S, Chen L, Krosting J, Sekinger E, Zhang W, et al. A novel FMR1 PCR method for the routine detection of low abundance expanded alleles and full mutations in fragile $X$ syndrome. Clin Chem 2010;56:399-408.

5. Tassone F, Pan R, Amiri K, Taylor AK, Hagerman PJ. A rapid polymerase chain reaction-based screening method for identification of all expanded alleles of the fragile X (FMR1) gene in newborn and high-risk populations. J Mol Diagn 2008;10:43-9.

6. Maddalena A, Richards CS, McGinniss MJ, Brothman A, Desnick RJ Grier RE, et al. Technical standards and guidelines for fragile $\mathrm{X}$ : the first of a series of disease-specific supplements to the Standards and Guidelines for Clinical Genetics Laboratories of the American College of Medical Genetics. Quality Assurance Subcommittee of the Laboratory Practice Committee. Genet Med 2001;3:200-5.

7. Pesso R, Berkenstadt $M$, Cuckle $H$, Gak E, Peleg L, Frydman $M$, et al. Screening for fragile $X$ syndrome in women of reproductive age. Prenat Diagn 2000;20:611-4.

8. Toledano-Alhadef H, Basel-Vanagaite L, Magal N, Davidov B, Ehrlich $\mathrm{S}$, Drasinover $\mathrm{V}_{\text {, et }}$ al. Fragile- $\mathrm{X}$ carrier screening and the prevalence of premutation and full-mutation carriers in Israel. Am J Hum Genet 2001;69:351-60.

9. Berkenstadt M, Ries-Levavi L, Cuckle H, Peleg L, Barkai G. Preconceptional and prenatal screening for fragile $\mathrm{X}$ syndrome: experience with 40,000 tests. Prenat Diagn 2007;27:991-4.

10. Spence WC, Black SH, Fallon L, Maddalena A, Cummings E, MenapaceDrew $\mathrm{G}$, et al. Molecular fragile $\mathrm{X}$ screening in normal populations. Am J Med Genet 1996:64:181-3.

11. Cronister A, DiMaio M, Mahoney MJ, Donnenfeld AE, Hallam S. Fragile X 
syndrome carrier screening in the prenatal genetic counseling setting. Genet Med 2005;7:246-50.

12. Ryynänen $M$, Heinonen $S$, Makkonen $M$, Kajanoja $E_{1}$ Mannermaa $A$, Pertti K. Feasibility and acceptance of screening for fragile $X$ mutations in low-risk pregnancies. Eur J Hum Genet 1999;7:212-6.

13. Kallinen J, Marin K, Heinonen S, Mannermaa A, Palotie A \& Ryynänen M Wide scope prenatal diagnosis at Kuopio University Hospital 19971998: integration of gene tests and fetal karyotyping. Br J Obstet Gynaecol 2001;108: 505-9.

14. Hill MK, Archibald AD, Cohen J, Metcalfe SA. A systematic review of population screening for fragile X syndrome. Genet Med 2010;12:396410.

15. Otsuka S, Sakamoto $Y$, Siomi H, Itakura M, Yamamoto K, Matumoto $H$, et al. Fragile $X$ carrier screening and FMR1 allele distribution in the Japanese population. Brain Dev 2010;32:110-4.

16. Huang $K F$, Chen WY, Tsai $Y C$, Lin $C C$, Chen $S H$, Tseng $C Y$, et al. Original article pilot screening for fragile $X$ carrier in pregnant women of southern. Taiwan J Chin Med Assoc 2003;66:204-9.
17. Song KH, Kim GJ, Whang YJ, Choi SR, Lee SP, Whang BC, et al. Allele distribution of FMR1 gene in Korean women. Kor J Obstet Gynecol 2002:45:990-3.

18. Hill MK, Archibald AD, Cohen J, Metcalfe SA. A systematic review of population screening for fragile X syndrome. Genet Med 2010;12:396410.

19. Peprah E. Fragile X syndrome: the FMR1 CGG repeat distribution among world populations. Ann Hum Genet 2012;76:178-91.

20. Haddad LA, Mingroni-Netto RC, Vianna-Morgante AM, Pena SD. A PCR-based test suitable for screening for fragile $X$ syndrome among mentally retarded males. Hum Genet 1996;97:808-12.

21. Brown WT, Nolin S, Houck G Jr, Ding X, Glicksman A, Li SY, et al. Prenatal diagnosis and carrier screening for fragile X by PCR. Am J Med Genet 1996;64:191-5.

22. Chen L, Hadd A, Sah S, Filipovic-Sadic S, Krosting J, Sekinger E, et al. An information-rich CGG repeat primed PCR that detects the full range of fragile $X$ expanded alleles and minimizes the need for southern blot analysis. J Mol Diagn 2010;12:589-600. 